\title{
Los Gráficos Estadísticos en los Libros de Texto de Educación Primaria en Costa Rica
}

\section{Statistical Graphs in Costa Rica Textbooks for Primary Education}

\author{
Maynor Jiménez-Castro * \\ ORCID iD 0000-0001-7984-6866 \\ Pedro Arteaga** \\ ORCID iD 0000-0002-8347-7669 \\ Carmen Batanero ${ }^{* * *}$ \\ ORCID iD 0000-0002-4189-7139
}

\begin{abstract}
Resumen
El objetivo del trabajo fue analizar los gráficos estadísticos incorporados en las dos series de libros de texto utilizadas con más frecuencia en la educación primaria en Costa Rica. Se analizan el tipo de gráfico, su complejidad semiótica y el contexto de donde se toman los datos, así como el tipo de tarea planteada, el nivel de lectura requerido y el propósito del gráfico dentro de la tarea. Se observa la predominancia del gráfico de barras, el tercer nivel de complejidad semiótica (representar una distribución), segundo nivel de lectura (leer entre los datos), contexto laboral y escolar, tareas de lectura y cálculo y propósito de análisis. Se describen las diferencias en función del grado y entre las dos editoriales, destacando las coincidencias y diferencias con resultados de otros estudios sobre la presentación de los gráficos estadísticos en los libros de texto en España y Chile.
\end{abstract}

Palabras clave: Gráficos estadísticos. Libros de texto. Educación primaria.

\begin{abstract}
The aim of this work was to analyze the statistical graphs included in the two most frequently series of textbooks used in Costa Rica basic education. We analyze the type of graph, its semiotic complexity, and the data context, as well as the type of task, reading level required to complete the task and purpose of the graph within the task. We observed the predominance of bar graphs, third level of semiotic complexity (representing a distribution), second reading level (reading between the data), work and school context, reading and computation tasks and analysis purpose. We describe the differences in the various grades and between both editorials, as well as differences and coincidences with results of other textbook studies carried out in Spain and Chile.
\end{abstract}

Keywords: Statistical graphs. Textbooks. Primary education.

\footnotetext{
* Doctorando en Ciencias de la Educación de la Universidad de Granada (UGR), Granada, España. Profesor en la Universidad de Costa Rica (UCR), Guápiles, Limón, Costa Rica. Dirección postal: La Roca, 3, Jiménez Centro, Pococí, provincia Limón, Costa Rica, código postal: 70202. E-mail: maynorj@correo.ugr.es.

** Doctor en Didáctica de la Matemática por la Universidad de Granada (UGR), España. Profesor del Departamento de Didáctica de la Matemática de la Universidad de Granada (UGR), Granada, España. Dirección postal: Campus de Cartuja, 18071, Granada, España. E-mail: parteaga@ugr.es.

*** Doctora en Matemática por la Universidad de Granada (UGR), España. Catedrática de la Universidad de Granada (UGR), Granada, España. Dirección postal: Campus de Cartuja, 18071, Granada, España. E-mail: batanero@ugr.es.
} 


\section{Introducción}

El conocimiento y la competencia en el trabajo con gráficos estadísticos es, hoy día, parte de la cultura estadística necesaria a todo ciudadano responsable, que encuentra numerosos gráficos de interés social en los medios de comunicación y en su trabajo profesional (ENGEL, 2019). Dicha cultura estadística, según Watson (2006), consta de los siguientes componentes:

- Conocimiento básico de los conceptos estadísticos y probabilísticos, incluidos los gráficos estadísticos.

- Comprensión de los razonamientos y argumentos estadísticos cuando se presentan dentro de algún informe en los medios de comunicación o en el trabajo, muchas veces apoyados en gráficos.

- Actitud crítica que se asume al cuestionar argumentos que estén basados en evidencia estadística usualmente representada gráficamente.

La necesidad de cultura estadística y conocimiento de los gráficos estadísticos crece, actualmente, debido a la cantidad de datos de libre disposición para el ciudadano que proporcionan las oficinas de estadística nacionales e internacionales por hacer comprensibles sus estudios (RIDGWAY, 2016). Por otro lado, los gráficos estadísticos facilitan la obtención de nueva información difícilmente visible en una lista desordenada de datos, por ejemplo, la moda, facilitando el razonamiento estadístico (WILD; PFANNKUCH, 1999).

En la actualidad, el Ministerio de Educación Pública de Costa Rica inicia una reforma educativa en el campo de la matemática (M.E.P, 2012), uno de cuyos objetivos es que los estudiantes adquieran una actitud crítica en relación a la información estadística publicada en los medios de comunicación. En consecuencia, en las directrices curriculares se ha dado un lugar relevante a la Estadística en todos los niveles del sistema educativo costarricense, siguiendo la tendencia internacional (CCSSI, 2010; MECD, 2014, NCTM, 2000).

En este trabajo nos centramos en los gráficos estadísticos, cuya comprensión, como se ha indicado, reviste especial importancia para alcanzar una cultura estadística básica (GAL, 2002) y es un componente del sentido estadístico (BATANERO, 2013). Cazorla (2002), por su parte, señala que son un instrumento potente para comunicar y resumir la información de los estudios estadísticos.

Antes de la reforma educativa matemática implementada en Costa Rica, en el año 2012, la educación estadística era tratada de manera tímida y superficial en el currículo matemático de secundaria, y omitida de la educación primaria. En las nuevas orientaciones los 
gráficos estadísticos se incluyen en el currículo costarricense en la Educación Primaria, con grados de $1^{\mathrm{o}}$ a $6^{\mathrm{o}}$, que corresponde a estudiantes entre 6 y 11 años, de la siguiente forma (M.E.P, 2012):

- En segundo grado, se introducen la lectura y construcción de los gráficos de barras. El objetivo principal es que el estudiante haga una lectura simple de la información y se genere la capacidad de extraer información de esas representaciones.

- Para el tercer año escolar, la propuesta curricular se concentra en la resolución de problemas, donde se espera que el estudiante adquiera la capacidad de formular interrogantes y proponer estrategias para la recolección y el resumen de los datos.

- En el cuarto año, el estudiante debe interpretar información en diagramas de puntos y se propone incorporar la hoja de cálculo para su construcción. En este sentido, también se espera que el estudiante sea capaz de identificar posibles errores en los datos recolectados. Adicionalmente, se incorporan las medidas de tendencia central como la media aritmética, la moda, el máximo y el mínimo, así como el recorrido de los datos como medida de dispersión.

- En el quinto año se incluyen los gráficos circulares aunque se recomienda su uso sólo para que el estudiante desarrolle la capacidad de una lectura crítica de la información y no para su construcción.

- En sexto se introducen los diagramas lineales para visualizar las tendencias en series de tiempo. También se contempla la comparación gráfica de dos o más grupos de datos.

Para poder tener éxito en la formación de los alumnos, es importante que el profesor conozca las características de las tareas propuestas en los libros de texto, en su país, y las pueda comparar con las de otros sistemas educativos.

El objetivo del trabajo es analizar la forma en que se consideran los gráficos en algunas series de libros de texto de Educación Primaria en Costa Rica, en los diferentes niveles educativos. En concreto, se pretende analizar el tipo de gráfico propuesto, su nivel de complejidad semiótica y contextos utilizados. Además, se estudian el tipo de tarea propuesta, el nivel de lectura y el propósito del gráfico considerado en las mismas. Nos centramos en el libro de texto, por su relevancia como recurso educativo y por constituir un paso intermedio entre las directrices curriculares y el currículo implementado en el aula (HERBEL, 2007). Completamos, de este modo, otros estudios sobre la misma temática en otros países, que se describen en la Sección 2. 


\section{Fundamentos teóricos}

Nos apoyamos en las investigaciones que describen la comprensión gráfica, los niveles de lectura y de complejidad de los gráficos y otras que analizan los gráficos estadísticos en libros de texto.

\subsection{Comprensión gráfica, niveles de lectura y complejidad semiótica}

A pesar de su aparente sencillez, Bertin (1967) indica que la lectura de un gráfico es una actividad compleja que conlleva tres procesos: el primero de ellos consiste en la interpretación de sus componentes (identificación externa), como el título y las etiquetas que remiten al tema del gráfico y variables representadas. Seguidamente, la identificación interna permite determinar los rangos de variación de las variables y las escalas representadas, por último, la percepción de la correspondencia entre los elementos identificados anteriormente y el contexto, sirven para obtener conclusiones sobre las variables y la realidad representada.

En base a esta teoría, algunos investigadores han propuesto jerarquías para evaluar diferentes niveles de dificultad en la lectura de un gráfico. En nuestro trabajo, consideramos los propuestos por Curcio (1987), Friel, Curcio y Bright (2001) y Shaughnessy, Garfield y Greer (1996), que son los siguientes:

1. Leer los datos - describe la capacidad específica de lectura literal del gráfico sin interpretar la información contenida en él. Un ejemplo sería la lectura de la frecuencia que corresponde a un valor de la variable.

2. Leer entre los datos - cuando se precisa comparar valores de los datos o aplicar cálculos a los mismos. Necesitaríamos este nivel para determinar la moda de una distribución.

3. Leer más allá de los datos - se refiere a la capacidad de inferir informaciones que no se reflejan directamente en el gráfico, por ejemplo, obtener el valor de un punto no representado en un diagrama de dispersión.

4. Leer detrás de los datos - consiste en realizar una valoración crítica con respecto al contenido del gráfico, por ejemplo, juzgar método de recolección de datos, o las conclusiones realizadas.

Adicionalmente, para valorar la complejidad semiótica de un Arteaga y Batanero (2011) y Batanero, Arteaga y Ruiz (2010) definen los siguientes niveles:

1. Representa solo algunos datos aislados, cuando en el gráfico no se considera todo el 
conjunto de datos, sino sólo algunos de sus valores.

2. Representación uno a uno de un conjunto de datos, en este nivel se representan todos los valores de las variables en el orden que se han dado, sin agrupar los datos del mismo valor ni calcular la frecuencia o la distribución de frecuencias. El orden de presentación de los valores de los datos es arbitrario y no corresponde al orden numérico.

3. Representación de la distribución de frecuencias de una única variable, considerando las ideas de variable, frecuencia y distribución. En este gráfico podrían visualizarse no sólo la variabilidad, sino también algunas medidas de posición central.

4. Representación de dos o más distribuciones sobre un mismo gráfico: en estos gráficos se representan varias distribuciones utilizando la misma escala, lo que supone analizar el rango de variación de cada una de las variables.

\subsection{Antecedentes de nuestro trabajo}

Un segundo referente son las investigaciones que han analizado los gráficos estadísticos, de donde obtendremos algunas de las variables analizadas y nos servirán de punto de comparación de nuestros resultados. Uno de los primeros estudios de este tipo es debido a Lemos (2006), quien analiza tres series de libros de texto de los cursos $1^{\mathrm{o}}$ a $4^{\mathrm{o}}$ de Educación Primaria en Brasil, estudiando el tipo de gráfico y la actividad pedida al alumno. Los gráficos se reducen casi por completo al diagrama de barras y las actividades principales son la lectura literal y la realización de cálculos a partir de los datos del gráfico.

Bivar y Selva (2011) investigan las actividades planteadas sobre los gráficos estadísticos en cinco colecciones de libros de texto para la Educación Primaria en Brasil, encontrando un predominio de las actividades consistentes en interpretar o completar gráficos, seguida por las de traducir un gráfico a otro.

Arteaga (2011) analiza las dos variables anteriores (tipo de gráfico y actividad pedida) en una serie completa de libros de texto españoles de Educación Primaria, a las que añade el nivel de lectura requerido en la editorial y el nivel de complejidad semiótica del gráfico. Todas estas variables son luego retomadas por Díaz-Levicoy en diversos trabajos, entre ellos Díaz-Levicoy y Arteaga (2014), Díaz-Levicoy, Giacomone y Arteaga (2017) y el que analizamos a continuación.

El principal trabajo que nos sirve de base es el realizado por Díaz-Levicoy et al. (2016), que analizan los gráficos estadísticos incluidos en tres series de libros de texto 
españoles y otras tres series chilenas de Educación primaria, y comparan con las orientaciones curriculares. Los autores estudian las mismas variables consideradas por por Arteaga (2011), es decir, el tipo de gráfico propuesto, su nivel de complejidad semiótica, la actividad que se pide al niño, y los niveles de lectura implícitos en la actividad. Concluyen la mayor presencia del gráfico de barras, con poco peso de otros tratados en el currículo. Los niveles de lectura más frecuentes fueron los intermedios, en la clasificación de Curcio (1989). Estos autores analizaron las mismas variables en libros de matemáticas en otros contextos: libros chilenos de $7^{\circ}$ nivel en Chile (DÍAZ-LEVICOY; ARTEAGA, 2014) y libros de matemática de Educación Primaria en Argentina (DÍAZ-LEVICOY; GIACOMONE; ARTEAGA, 2017) obteniendo resultados muy similares.

Otros autores han analizado las mismas variables en pruebas de evaluación externas de los estudiantes. Así, Castellanos (2013) analiza los gráficos estadísticos incluidos en tres años de aplicación de la Prueba SABER, en Colombia, para estudiantes de quinto año de primaria. La autora informa que la mayoría de los gráficos presentados en estas pruebas son gráficos de barras, el segundo nivel de lectura, leer dentro los datos, y el nivel 3 de complejidad semiótica. Los ítems relacionados con gráficos incluyen, generalmente, más de una actividad, siendo la más frecuente la lectura del gráfico, seguida del cálculo y las comparaciones de datos.

Mingorance (2014) analiza los gráficos y tablas estadísticas en las pruebas de diagnóstico andaluzas obligatorias para los niños de $4^{\circ}$ grado, donde la mitad de los gráficos propuestos corresponden a diagrama de barras y el resto son pictogramas, cartogramas, gráfico de sectores o de líneas. También, analiza los contextos siguiendo el modelo PISA, que son generalmente personales y sociales. El nivel de lectura es igualmente intermedio (niveles 2 y 3 en la clasificación de Curcio).

\section{Metodología}

La muestra estuvo formada por las dos series de libros de texto más utilizadas en la educación primaria de Costa Rica, en ediciones actualizadas, que atienden a las nuevas políticas curriculares de la reforma matemática implementada en el año 2012: Asociación Libros para Todos del Grupo Nación, que representamos en nuestro estudio por (AL) y Editorial Santillana, que se indica por (S). En estos libros se llevó a cabo un análisis de contenido, que permite identificar, de manera sistemática y objetiva, características específicas de un texto. Se lleva a cabo a través de las siguientes fases (PORTA; SILVA, 
2003):

- Determinar el objeto o tema de análisis: en cada libro de texto se estudian las actividades que incorporan gráficos estadísticos.

- Determinar las reglas de codificación y categorías: se procede a identificar a priori a partir de investigaciones previas las categorías sobre tipo de gráfico, complejidad semiótica, tareas propuestas en los libros de texto, propósitos y contexto.

- Comprobar la fiabilidad del sistema de codificación-categorización: para lo cual la codificación fue revisada por varias personas.

En estos libros de texto se realizaron dos tipos de análisis: El primero de ellos consistió en el análisis de todos los gráficos estadísticos encontrados, que fueron en total 167 y de los cuáles se estudiaron tres variables:

- Tipo de gráfico. Se consideran todos los citados en las directrices curriculares y cualquiera que aparezca en los textos analizados.

- Niveles de complejidad semiótica definido por Arteaga y colaboradores (ARTEAGA, 2011; BATANERO; ARTEAGA; RUIZ, 2010).

- Contexto del gráfico. Esta variable responde a la clasificación establecida en los estudios PISA, que se describe en OECD (2016).

Puesto que, en relación con cada gráfico encontrado, se propone, generalmente, más de una actividad, a continuación se analizó independientemente cada una de las actividades propuestas para cada gráfico (en total 398 actividades), en las que se estudiaron las siguientes variables adicionales:

- Niveles de lectura. Nivel de lectura del gráfico que la ejecución de la actividad propuesta requiere por parte del estudiante, considerando los aportes de Curcio y colaboradores (CURCIO, 1989; FRIEL; CURCIO; BRIGHT, 2001; SHAUGHNESSY; GARFIELD; GREER, 1996).

- Tipo de actividad. Donde se han considerado las siguientes: leer, completar, calcular, construir, ejemplificar, traducir a otra representación e inventar problema; que se describen y ejemplifican en el apartado de resultados y análisis.

- Propósito del gráfico dentro de la actividad: Siguiendo a Kosslyn (1985), hemos considerado los siguientes: análisis, comunicación y meramente constructivo. 


\section{Resultados del análisis de los gráficos}

En esta sección se muestran los principales resultados obtenidos, que se comparan con los obtenidos en otras investigaciones previas.

\subsection{Tipos de gráficos}

Al clasificar los gráficos propuestos en los libros de texto, en las dos editoriales, se han encontrado gráficos de barras y de líneas simples y múltiples, pictogramas, diagramas de puntos y gráficos de sectores (Tabla 1). Se observa el predominio del gráfico de barras, lo que coincide con las investigaciones de Lemos (2006), Arteaga (2011), Castellanos (2013) y Mingorance (2014). En Díaz-Levicoy et al. (2016) los gráficos de barras representan el 46\% de las actividades en los textos españoles y el $42,5 \%$ de los chilenos.

Tabla 1 - Distribución del tipo de gráfico en cada editorial

\begin{tabular}{|c|c|c|c|c|}
\hline \multirow{3}{*}{ Tipo de gráfico } & \multicolumn{4}{|c|}{ Editorial } \\
\hline & \multicolumn{3}{|c|}{$\mathbf{A L}$} & $\mathbf{S}$ \\
\hline & $\mathbf{N}$ & $\%$ & $\mathbf{N}$ & $\%$ \\
\hline Barras & 31 & 55,4 & 63 & 56,8 \\
\hline Líneas & 6 & 10,7 & 6 & 5,4 \\
\hline Pictogramas & & & 17 & 15,3 \\
\hline Sectores & 7 & 12,5 & 8 & 7,2 \\
\hline Diagrama de puntos & 9 & 16,1 & 9 & 8,1 \\
\hline Líneas múltiples & 2 & 3,6 & 1 & 0,9 \\
\hline Barras múltiples & 1 & 1,8 & 7 & 6,3 \\
\hline Total & 56 & 100 & 111 & 100 \\
\hline
\end{tabular}

Los siguientes gráficos más utilizado en estas editoriales son los pictogramas y diagramas de puntos, seguidos por los gráficos de sectores, mientras en el estudio de DíazLevicoy et al. (2016) el segundo gráfico más usado fue el líneal (20\%), en los textos españoles, y el pictograma $(22,6 \%)$, en los chilenos.

Tanto las orientaciones curriculares españolas (MECD, 2014), como las costarricenses (M.E.P, 2012), recomiendan los gráficos de barras, pictogramas y gráficos de sectores, como las principales gráficas en la educación primaria. Adicionalmente, el currículo básico español, incluye polígonos de frecuencias y la pirámide poblacional, que no son utilizados en el contexto costarricense. En el caso de Chile, el currículo escolar de primaria (MINEDUC, 2012), incluye diagramas de puntos líneas y diagramas de tallo y hojas.

En cuanto a la distribución del tipo de gráfico según el año escolar (Tabla 2) las series 
analizadas incorporan mayor variedad de gráficos conforme se avanza el curso. En primer año, sólo se presenta el gráfico de barras, en segundo y tercero se utilizan además los pictogramas, en cuarto se agregan los gráficos de líneas y en quinto los gráficos circulares, mientras que en sexto año, se incluyen gráficos de barras dobles, de puntos y líneas múltiples.

No obstante, predomina el uso de gráficos de barras (y sus variantes) en los todos los niveles, excepto el cuarto curso. En el estudio de Díaz-Levicoy et al. (2016), en los dos primeros cursos aparecen diagramas de barras y pictogramas en los textos chilenos, pero los españoles se limitan a gráficos de barras. En los textos chilenos del tercer a quinto curso, los gráficos de línea son el segundo tipo más frecuente, mientras que en los libros costarricenses esta representación gráfica se utiliza muy poco. En los dos países se incorporan gráficos de líneas, pictogramas y sectores, a partir del cuarto curso, mientras que en nuestro estudio estas representaciones gráficas solo se proponen en el sexto año.

Tabla 2 - Distribución de gráficos por año escolar

\begin{tabular}{|c|c|c|c|c|c|c|c|c|c|c|c|c|}
\hline \multirow{3}{*}{ Tipo de gráfico } & \multicolumn{12}{|c|}{ Año } \\
\hline & \multicolumn{2}{|c|}{$1^{\circ}$} & \multicolumn{2}{|c|}{$2^{\circ}$} & \multicolumn{2}{|c|}{$3^{\circ}$} & \multicolumn{2}{|c|}{$4^{\circ}$} & \multicolumn{2}{|c|}{$5^{\circ}$} & \multicolumn{2}{|c|}{$6^{\circ}$} \\
\hline & $\mathbf{N}$ & $\%$ & $\mathbf{N}$ & $\%$ & $\mathbf{N}$ & $\%$ & $\mathbf{N}$ & $\%$ & $\mathbf{N}$ & $\%$ & $\mathbf{N}$ & $\%$ \\
\hline Barras & 8 & 100 & 30 & 68,2 & 25 & 96,2 & 10 & 30,3 & 20 & 71,4 & 1 & 3,6 \\
\hline Líneas & & & & & & & 5 & 15,2 & & & 7 & 25 \\
\hline Pictogramas & & & 14 & 31,8 & 1 & 3,8 & 2 & 6,1 & & & & \\
\hline Sectores & & & & & & & & & 8 & 28,6 & 7 & 25 \\
\hline Diagramas de puntos & & & & & & & 16 & 48,5 & 0 & 0 & 2 & 7,1 \\
\hline Líneas múltiples & & & & & & & & & & & 3 & 10,7 \\
\hline Barras múltiples & & & & & & & & & & & 8 & 28,6 \\
\hline Total & 8 & 100 & 44 & 100 & 26 & 100 & 33 & 100 & 28 & 100 & 28 & 100 \\
\hline
\end{tabular}

Solo el 4,7\% de las actividades analizadas se ubican en el primer año y la mayor frecuencia se concentra en segundo y cuarto año (26,3\% y 19,7\% respectivamente). Quinto y sexto grado mantienen una equivalencia en frecuencia con $16,3 \%$ de las actividades analizadas. Estos resultados difieren de los obtenidos en Díaz-Levicoy et al. (2016), donde la mayor cantidad de actividades que involucran gráficos estadísticos se concentran en los dos últimos cursos.

\subsection{Complejidad semiótica de los gráficos}

Esta variable se valora de acuerdo con la clasificación propuesta por Batanero, Arteaga y Ruiz (2010), tomando en cuenta los siguientes niveles:

N1. Representa solo sus resultados individuales: corresponde a la actividad semiótica 
de más bajo nivel, que consiste en representar solo datos aislados, olvidando el resto. No se han encontrado actividades en este nivel.

N2. Representación de un conjunto de datos uno a uno: en este nivel de complejidad no se forma la distribución de datos. Es posible la extracción de datos al visualizarse todos los valores de las variables, pero no lo suficiente para llegar a la extracción de tendencias. Un ejemplo se muestra en la Figura 1, donde se grafica un listado de datos sin formar su distribución de frecuencias.

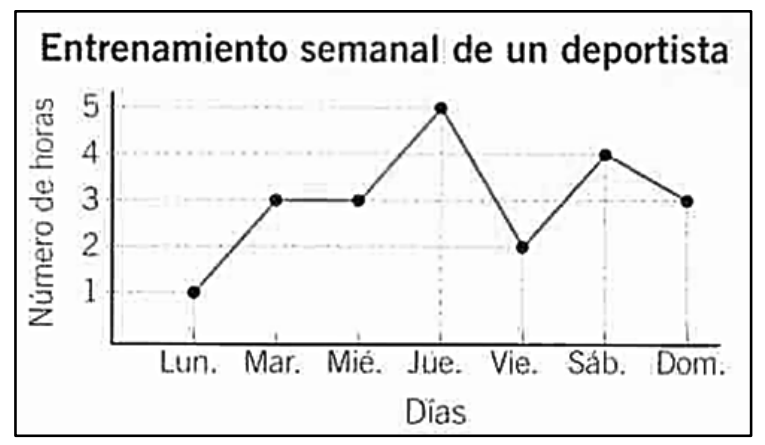

Figura 1 - Ejemplo de complejidad semiótica de nivel 2 Fuente: Tomado de AL4, p. 278

N3. Representación de una única distribución de frecuencias: en este nivel de complejidad semiótica, se resume una sola distribución de datos, lo que permite identificar medidas de tendencia central como moda y mediana. Un ejemplo de este tipo de complejidad semiótica se puede observar en la Figura 2.

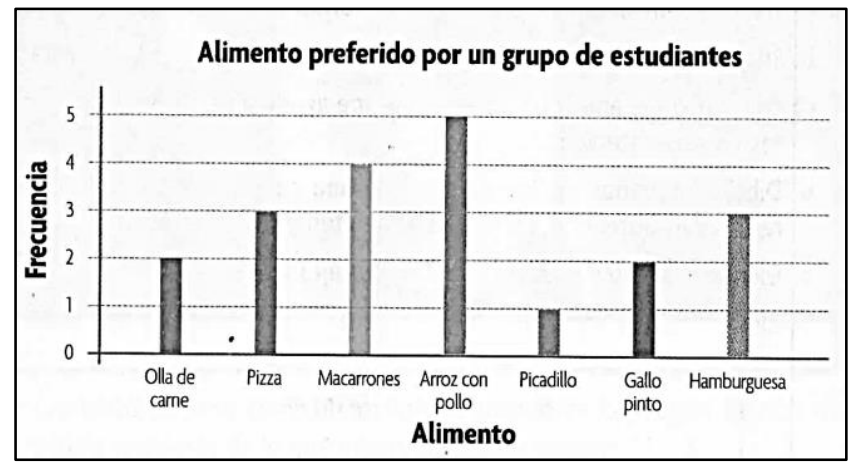

Figura 2 - Ejemplo de complejidad semiótica 3, representación de una distribución de datos Fuente: Tomado de AL5, p. 146

N4. Representación de dos distribuciones sobre un mismo gráfico: este es el más alto nivel de complejidad semiótica establecido para un gráfico gráfico (ARTEAGA 2011; BATANERO; ARTEAGA; RUIZ, 2010). Un ejemplo de este nivel de complejidad se muestra en la Figura 3, en la que se visualizan dos series de datos que representan los pasatiempos preferidos de niños y adultos. 


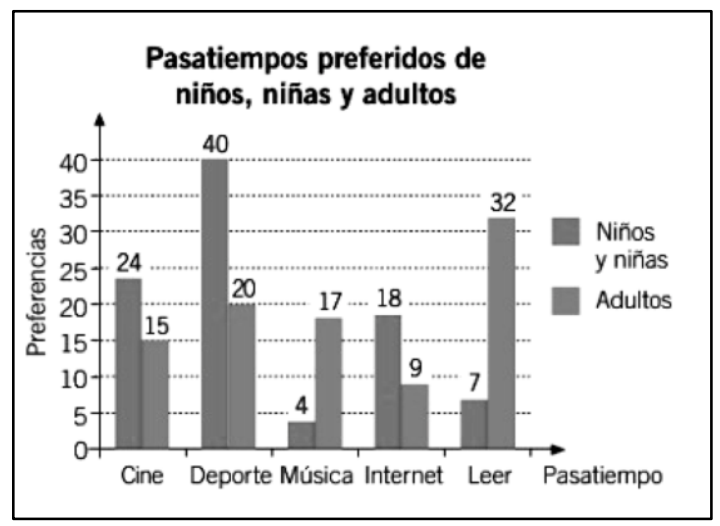

Figura 3 - Ejemplo de complejidad semiótica 4: representación de dos distribuciones Fuente: Tomado de S6, p. 263

Como se puede observar en la Tabla 3, la mayoría de los gráficos corresponden al nivel 3, representación de una distribución, siguiendo los niveles 2 y 4, proponiendo la editorial S comparativamente más gráficos de nivel N2. Los datos generales coinciden con los resultados de Díaz-Levicoy et al. (2016), donde el nivel 3 se encuentra en el 58,6\% en los textos españoles y $66,1 \%$ en los chilenos. El segundo nivel semiótico más frecuente, planteado en el estudio español, fue el nivel 4 (22,3\%) que sólo representa el 8,4\% de gráficos en Costa Rica, mientras el nivel 2 aparece con $24,0 \%$ y $27 \%$ respectivamente en España y Chile.

Tabla 3 - Frecuencia y porcentaje de gráficos por nivel de complejidad y editorial

\begin{tabular}{|c|c|c|c|c|}
\hline \multirow{3}{*}{$\begin{array}{c}\text { Nivel de } \\
\text { Complejidad } \\
\end{array}$} & \multicolumn{4}{|c|}{ Editorial } \\
\hline & \multicolumn{2}{|c|}{$\mathbf{A L}$} & \multicolumn{2}{|c|}{$\mathbf{S}$} \\
\hline & $\mathrm{N}$ & $\%$ & $\mathrm{~N}$ & $\%$ \\
\hline N2 & 6 & 10,7 & 34 & 30,6 \\
\hline N3 & 44 & 78,6 & 69 & 62,2 \\
\hline N4 & 6 & 10,7 & 8 & 7,2 \\
\hline Total & 56 & 100 & 111 & 100 \\
\hline
\end{tabular}

La Tabla 4 muestra el incremento del nivel de complejidad de los gráficos conforme se avanza en el nivel escolar. En primer año solo se encontraron actividades del nivel 2, mientras que en sexto año se encontraron actividades de todos los niveles de complejidad. De $2^{\circ}$ al $5^{\circ}$ grado predominan las actividades del nivel 3 y solo en el $6^{\circ}$ año las de nivel 4. En el estudio de Díaz-Levicoy et al. (2016), encontramos en los textos españoles actividades de nivel de complejidad semiótica de nivel 4 a partir del segundo curso, predominando este nivel en el quinto y sexto curso. Por el contrario, al igual que en Costa Rica, estos gráficos fueron poco frecuentes en los textos chilenos donde este nivel de complejidad sólo aparece en los cursos quinto y sexto. 
Tabla 4 - Frecuencia y porcentaje de gráficos por nivel de complejidad y año escolar

\begin{tabular}{|c|c|c|c|c|c|c|c|c|c|c|c|c|}
\hline \multirow{3}{*}{$\begin{array}{c}\text { Nivel de } \\
\text { Complejidad }\end{array}$} & \multicolumn{12}{|c|}{ Año Escolar } \\
\hline & \multicolumn{2}{|c|}{$1^{\circ}$} & \multicolumn{2}{|c|}{$2^{\circ}$} & \multicolumn{2}{|c|}{$3^{\circ}$} & \multicolumn{2}{|c|}{$4^{\circ}$} & \multicolumn{2}{|c|}{$5^{\circ}$} & \multicolumn{2}{|c|}{$6^{\circ}$} \\
\hline & $\mathrm{N}$ & $\%$ & $\mathrm{~N}$ & $\%$ & $\mathrm{~N}$ & $\%$ & $\mathrm{~N}$ & $\%$ & $\mathrm{~N}$ & $\%$ & $\mathrm{~N}$ & $\%$ \\
\hline $\mathrm{N} 2$ & 8 & 100 & 14 & 31,8 & & & 6 & 18,2 & 4 & 14,3 & 8 & 28,6 \\
\hline N3 & & & 30 & 68,2 & 26 & 100 & 25 & 75,8 & 24 & 85,7 & 8 & 28,6 \\
\hline N4 & & & & & & & 2 & 6,1 & & & 12 & 42,9 \\
\hline Total & 8 & 100 & 44 & 100 & 26 & 100 & 33 & 100 & 28 & 100 & 28 & 100 \\
\hline
\end{tabular}

\subsection{Contextos presentados}

Se analizó el contexto del gráfico, utilizando la clasificación establecida en los estudios PISA (OECD, 2016), donde se sugiere que el rendimiento matemático de un individuo puede ser influenciada por el contexto en el que desarrollen los problemas, que, por consiguiente, puede afectar la comprensión del gráfico estadístico. Esta clasificación contempla los siguientes contextos:

Contexto personal: corresponde a situaciones en el ámbito de actividades personales del niño, de su familia o grupo de iguales. Un ejemplo de este contexto se presenta en la Figura 1, donde el gráfico muestra el número de horas semanal que dedica un deportista a su entrenamiento.

Contexto social: corresponden a los problemas donde se aborda aspectos del ámbito social, más allá de la vida personal del niño; por ejemplo, el barrio, o ciudad. Pueden corresponder a sistemas de votación, transporte público, gobierno, políticas públicas, demografía, publicidad, estadísticas nacionales o economía. También se incluyen en este contexto los juegos de azar, por ser ampliamente utilizados en la sociedad. Un ejemplo se muestra en la Figura 3, donde se describen los pasatiempos preferidos de niños y adultos.

C3. Contexto laboral o escolar: consisten en aquellas situaciones donde el problema aborda aspectos del mundo laboral: escuela, trabajo, empleo, producción, venta etc. Vemos un ejemplo en la Figura 4 que muestra datos de producción de un cierto producto (cajas de fresas).

C4. Contexto científico: este contexto se relaciona con problemáticas del ámbito de la ciencia y la tecnología, e incluye aspectos como: el clima, ecología, medicina, ciencia espacial, genética, medición y el mundo de las matemáticas en sí. También se incluyen en esta categoría los contextos relacionados con la vida del niño en la escuela. 


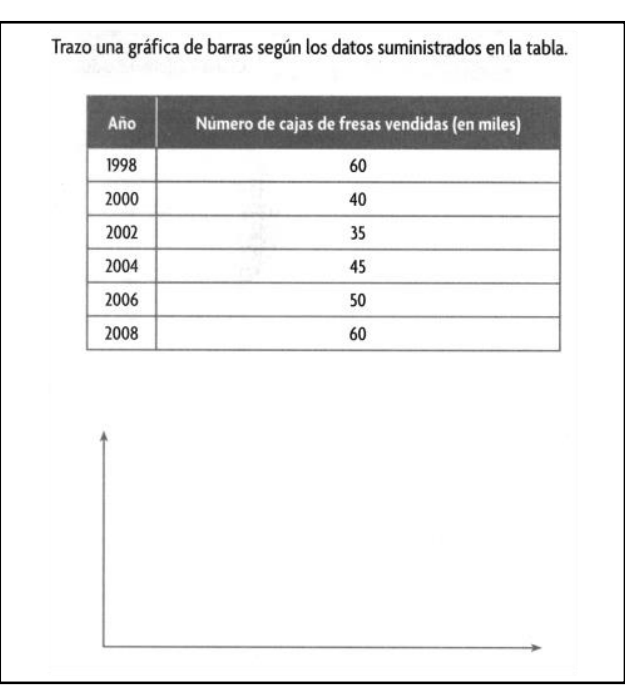

Figura 4 - Ejemplo de construcción de un gráfico ubicado en $5^{\circ}$ año.

Fuente: tomado de AL5, p. 150

Los datos sobre el contexto se resumen en la Tabla 5, donde se observa que el contexto utilizado en $\mathrm{Al}$ es primordialmente el laboral/escolar $(48,2 \%)$, mientras en $\mathrm{S}$ tanto este contexto como el personal aparecen en el 33,3\%, de las actividades. El personal es el menos abordado en $\mathrm{Al}$ (3,5\%). Ambas editoriales apuntan fuertemente al contexto escolar/laboral y en menor medida al científico.

Tabla 5 - Frecuencia y porcentaje de gráficos por tipo de contexto y editorial

\begin{tabular}{|c|c|c|c|c|}
\hline \multirow{2}{*}{ Contexto } & \multicolumn{2}{|c|}{$\mathbf{A L}$} & \multicolumn{2}{|c|}{$\mathbf{S}$} \\
\hline & $\mathbf{N}$ & $\%$ & $\mathbf{N}$ & $\%$ \\
\hline Personal & 2 & 3,6 & 37 & 33,3 \\
\hline Social & 16 & 28,6 & 21 & 18,9 \\
\hline Escolar & 27 & 48,2 & 37 & 33,3 \\
\hline Científico & 11 & 19,6 & 16 & 14,4 \\
\hline Total & 56 & 100 & 111 & 100 \\
\hline
\end{tabular}

La relación del contexto con el nivel educativo, se muestra en la Tabla 6, donde el contexto laboral/ escolar domina los niveles del $2^{\circ}$ al $5^{\circ}$ año, sobre todo debido a situaciones relacionadas con el trabajo en la escuela.

Tabla 6 - Frecuencia y porcentaje de gráficos por tipo de contexto y año escolar

\begin{tabular}{|c|c|c|c|c|c|c|c|c|c|c|c|c|}
\hline \multirow{3}{*}{ Tipo de contexto } & \multicolumn{12}{|c|}{ Año Escolar } \\
\hline & \multicolumn{2}{|c|}{$\mathbf{1}^{\circ}$} & \multicolumn{2}{|c|}{$2^{\circ}$} & \multicolumn{2}{|c|}{$3^{\circ}$} & \multicolumn{2}{|c|}{$4^{\circ}$} & \multicolumn{2}{|c|}{$5^{\circ}$} & \multicolumn{2}{|c|}{$6^{\circ}$} \\
\hline & $\mathbf{N}$ & $\%$ & $\mathbf{N}$ & $\%$ & $\mathbf{N}$ & $\%$ & $\mathbf{N}$ & $\%$ & $\mathbf{N}$ & $\%$ & $\mathbf{N}$ & $\%$ \\
\hline Personal & 3 & 37,8 & 14 & 31,8 & 7 & 26,9 & 7 & 21,2 & 2 & 7,1 & 6 & 21,4 \\
\hline Social & 3 & 37,5 & 8 & 18,2 & 9 & 34,6 & 7 & 21,2 & 2 & 7,1 & 8 & 28,6 \\
\hline Escolar & 2 & 25 & 17 & 38,6 & 9 & 34,6 & 12 & 36,4 & 18 & 64,3 & 6 & 21,4 \\
\hline Científico & & & 5 & 11,4 & 1 & 3,8 & 7 & 21,2 & 6 & 21,4 & 8 & 28,6 \\
\hline Total & 8 & 100 & 44 & 100 & 26 & 100 & 33 & 100 & 28 & 100 & 28 & 100 \\
\hline
\end{tabular}

En primer año son más frecuentes los contextos personal y social $(37,8 \%)$, coincidiendo 
con la investigación de Mingorance (2014), mientras que el sexto predominan los contextos social y científico con un $28,6 \%$. Dicho contexto científico es escaso en los primeros años escolares, pero aumenta a partir del cuarto grado.

\section{Resultados del análisis de las actividades propuestas para cada gráfico}

\subsection{Nivel de lectura de los gráficos}

Se analizó el nivel de lectura requerido en las actividades propuestas, utilizando la clasificación de Curcio (1987), Shaughnessy, Garfield y Greer (1996) y Friel, Curcio y Bright (2001), donde se consideran cuatro niveles.

N1. Leer los datos: este nivel la actividad se concentra en responder preguntas sobre la frecuencia de un valor de una variable (o el valor al que corresponde una frecuencia), sin llegar a establecer otras conexiones ni a realizar otros cálculos con los datos. Un ejemplo se muestra en la Figura 5, donde se pide completar un gráfico de barras para representar la frecuencia de una serie de objetos (previamente clasificados) que se muestran en una imagen.

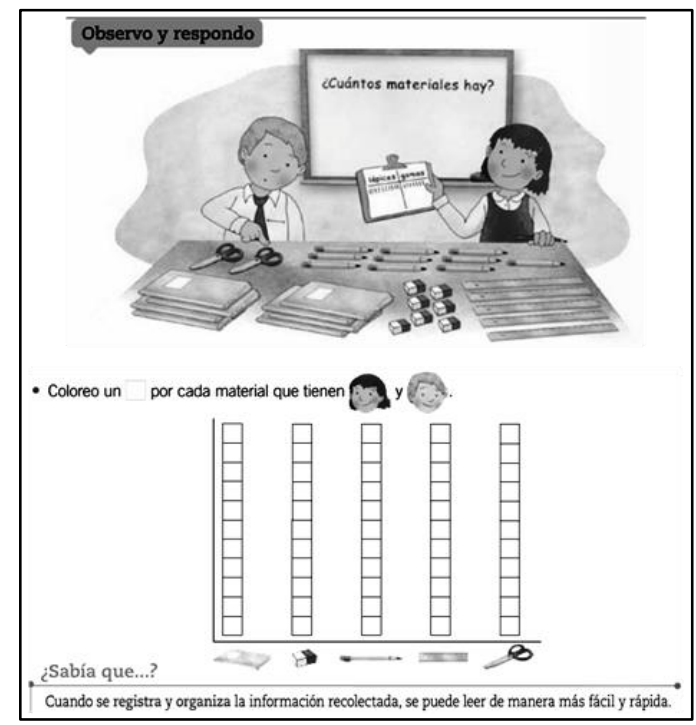

Figura 5 - Ejemplo de nivel de lectura N1 leer los datos Fuente: Tomado de S1, p. 298

N2. Leer dentro de los datos: este nivel se presenta cuando se deben realizar comparaciones u operaciones con los datos obtenidos de un gráfico para resolver la actividad. Involucra identificar relaciones entre los datos, como ocurre en las preguntas a y c de la actividad presentada en la Figura 6. En la primera de ellas se pide determinar la tendencia creciente o decreciente de la gráfica, lo que requiere la comparación de los datos en diferentes años, mientras que en la segunda deben compararse las dos gráficas entre sí. 


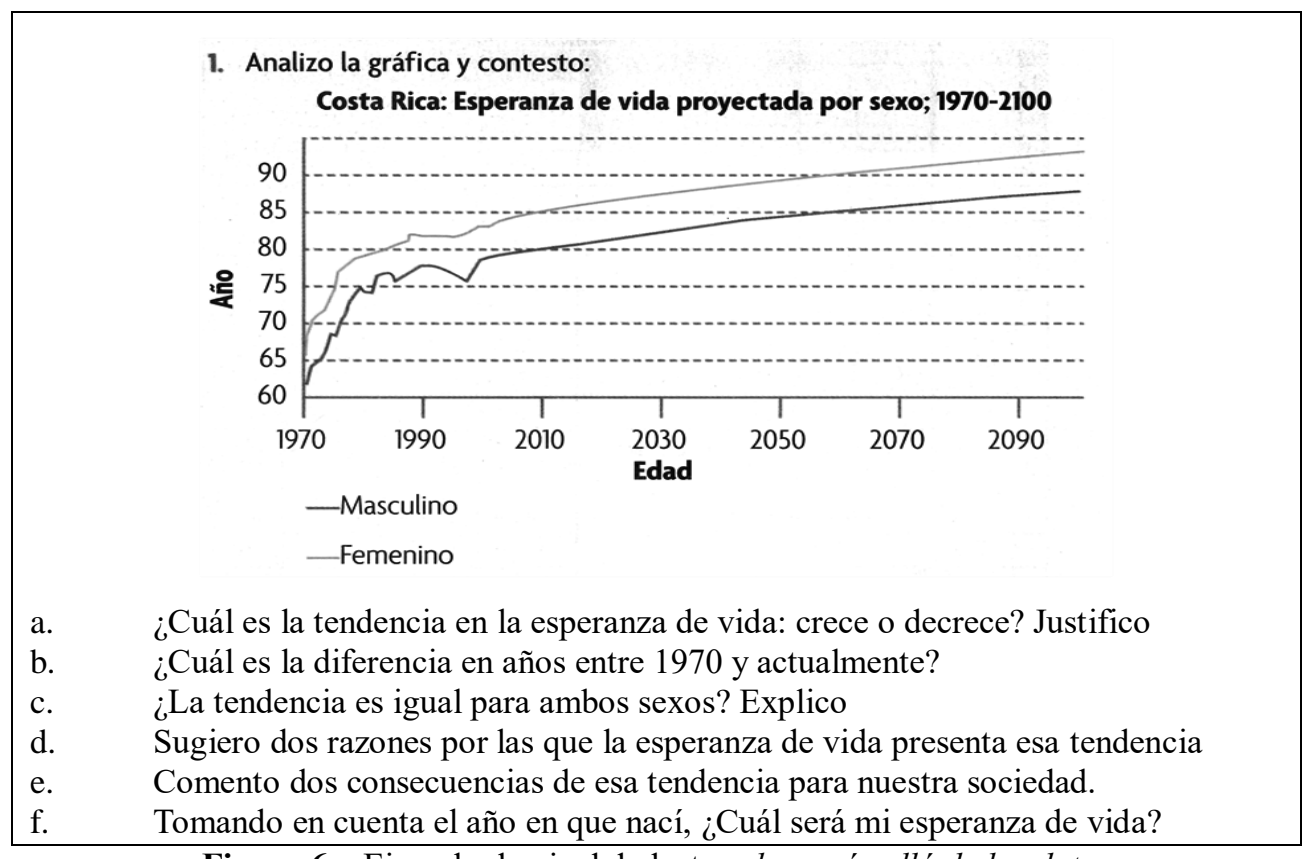

Figura 6 - Ejemplo de nivel de lectura leer más allá de los datos Fuente: reproducido de AL5 p. 166

N3. Leer más allá de los datos: este nivel de lectura se logra en actividades donde se pide obtener datos no representados explícitamente dentro del gráfico, es decir interpolar o extrapolar un dato en el gráfico. Así, en la Figura 5 la pregunta f corresponde a este nivel de lectura, pues dependiendo del año de nacimiento el estudiante ha de localizar este dato en la gráfica y, posiblemente, si el año no es múltiplo de 10 debe localizarlo en el eje X para luego identificar la esperanza de vida que corresponde a dicho año.

N4. Leer detrás de los datos. Consiste en realizar una valoración crítica con respecto al método de recolección de datos, validez y fiabilidad, que permita justificar las conclusiones realizadas. Si bien este nivel de lectura no es común en la educación primaria, lo consideramos en actividades como la presentada en la pregunta d de la Figura 5, donde se pide al estudiante que piense en algunas razones del aumento de la esperanza de vida.

En esta variable (Tabla 7), ambas editoriales evidencian un interés en promover una lectura sencilla de los datos en un gráfico, al proponer actividades de los niveles de lectura N1 Leer los datos (27,2\% y 34,3\%) y $\mathrm{N} 2$ Leer dentro de los datos (54,4\% y 46,2\%) principalmente. Esta misma situación se presenta en el estudio realizado por Díaz-Levicoy et al. (2016), donde resulta más frecuente el tipo de lectura N2 de leer dentro de los datos, tanto en Chile como en España; sin embargo, el porcentaje acumulado en los niveles N3 y N4 representan en Costa Rica un 19,5\%, mientras que en España y Chile solo el 6,1\% y el 7,4\%, respectivamente. 
Tabla 7 - Nivel de lectura y frecuencia en los libros de texto analizados

\begin{tabular}{|c|c|c|c|c|}
\hline \multirow{3}{*}{ Nivel de lectura } & \multicolumn{4}{|c|}{ Editorial } \\
\hline & \multicolumn{2}{|c|}{$\mathbf{A L}$} & \multicolumn{2}{|c|}{$\mathbf{S}$} \\
\hline & $\mathrm{N}$ & $\%$ & $\mathrm{~N}$ & $\%$ \\
\hline N1 & 40 & 27,2 & 86 & 34,3 \\
\hline N2 & 80 & 54,4 & 116 & 46,2 \\
\hline N3 & 13 & 8,8 & 34 & 13,5 \\
\hline $\mathrm{N} 4$ & 14 & 9,5 & 15 & 6 \\
\hline Total & 147 & 100 & 251 & 100 \\
\hline
\end{tabular}

La Tabla 8 muestra como el nivel de lectura se va incrementando conforme se avanza en el nivel escolar. Se inicia con niveles 1 y 2 en primer año y se incorporan los niveles 3 y 4 paulatinamente desde el segundo al sexto curso.

Tabla 8 - Frecuencia y porcentaje de gráficos por nivel de lectura y año escolar

\begin{tabular}{|c|c|c|c|c|c|c|c|c|c|c|c|c|}
\hline \multirow{3}{*}{ Nivel de Lectura } & \multicolumn{12}{|c|}{ Año Escolar } \\
\hline & \multicolumn{2}{|c|}{$1^{\circ}$} & \multicolumn{2}{|c|}{$2^{\circ}$} & \multicolumn{2}{|c|}{$3^{\circ}$} & \multicolumn{2}{|c|}{$4^{\circ}$} & \multicolumn{2}{|c|}{$5^{\circ}$} & \multicolumn{2}{|c|}{$6^{\circ}$} \\
\hline & $\mathbf{N}$ & $\%$ & $\mathbf{N}$ & $\%$ & $\mathbf{N}$ & $\%$ & $\mathbf{N}$ & $\%$ & $\mathbf{N}$ & $\%$ & $\mathbf{N}$ & $\%$ \\
\hline N1 & 21 & 65,6 & 33 & 37,5 & 28 & 50,9 & 21 & 25,9 & 12 & 17,6 & 11 & 14,9 \\
\hline $\mathrm{N} 2$ & 10 & 31,3 & 41 & 46,6 & 22 & 40 & 39 & 48,1 & 39 & 57,4 & 45 & 60,8 \\
\hline N3 & 1 & 3,1 & 7 & 8 & 4 & 7,3 & 16 & 19,8 & 12 & 17,6 & 7 & 9,5 \\
\hline N4 & & & 7 & 8 & 1 & 1,8 & 5 & 6,2 & 5 & 7,4 & 11 & 14,9 \\
\hline Total & 32 & 100 & 88 & 100 & 55 & 100 & 81 & 100 & 68 & 100 & 74 & 100 \\
\hline
\end{tabular}

Esta situación contrasta con lo reflejado en el estudio descrito por Díaz-Levicoy et al. (2016), donde los libros españoles y chilenos se mantienen en el nivel de lectura 1 en los dos primeros cursos y sube a partir del tercero (Chile) o cuarto curso (España). En Costa Rica, los niveles de lectura 3 y 4 aparecen significativamente a partir del segundo año y se incrementa su frecuencia hasta el sexto año. En el contexto español, el máximo nivel de lectura solo se presenta en el quinto curso, en cambio en el contexto chileno, se incorpora de manera sostenida desde el cuarto grado, coincidiendo con las recomendaciones dadas en NCTM (2000) de promover la realización de inferencias y la obtención de predicciones a partir de los datos analizados.

\subsection{Tipo de tarea requerida}

Las tareas que se plantean respecto a los gráficos estadísticos en los libros de texto en Costa Rica, se clasificaron en categorías según de los estudios de Arteaga (2011), Castellanos (2013) y Díaz-Levicoy et al. (2016) y se describen a continuación.

Leer el gráfico: cuando se pide responder a preguntas sobre los datos explícitamente 
representados en un gráfico; por ejemplo leer el título o etiquetas de las escalas, describir las variables representadas u obtener la frecuencia de un valor de la variable. Las preguntas propuestas en la Figura 6 serían ejemplos de actividad de lectura.

Completar: En este caso la tarea consiste en completar o bien modificar la representación de datos en un gráfico que se encuentra parcialmente construido. Un ejemplo se muestra en la Figura 7, en que se pide pintar casillas para completar el gráfico construido.

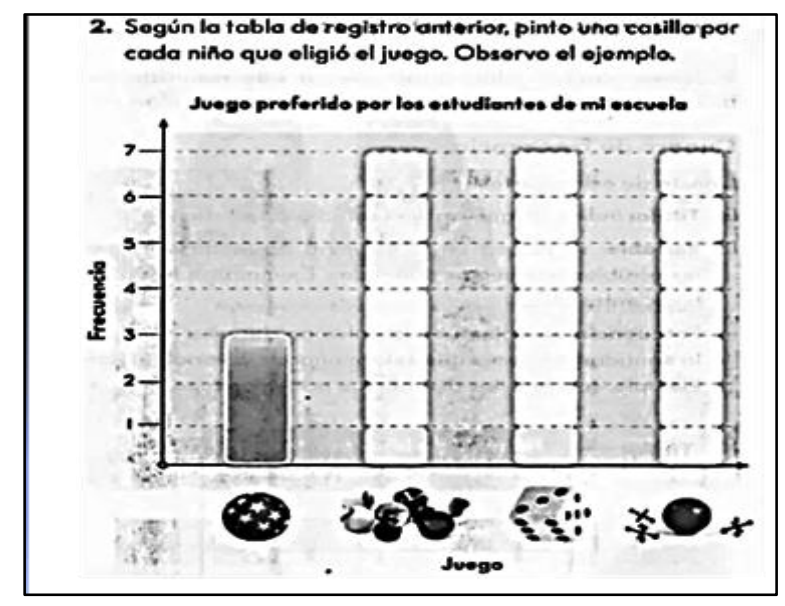

Figura 7 - Ejemplo de tarea completar Fuente: tomado de AL2, p. 176

Calcular: en este tipo de tarea se clasifican todas aquellas actividades en las que el estudiante debe responder a preguntas sobre los datos literales $\mathrm{y}$, además, requieran de algunos cálculos sencillos, como pueden ser: sumar o restar, calcular la media, moda, mediana y rango. Este tipo de tarea se puede observar en la pregunta b de la Figura 5.

Construir: en este caso se pide al estudiante que construya un gráfico con los datos que se les proporciona. Un ejemplo se muestra en la Figura 4, donde se proporciona una lista de datos y se pide construir el gráfico del cual sólo se proporcionan los ejes. El estudiante deberá fijar el título, escala y elementos de dicho gráfico.

Ejemplificar: en este tipo de actividad se utiliza el gráfico sólo para efectos de explicar su estructura o bien para orientar la construcción de un gráfico y sus principales elementos.

Traducir a otra representación: en algunas actividades se solicita elaborar la tabla de frecuencias que corresponde a los datos representados en un gráfico o bien, utilizar otra representación para pasar de un tipo de gráfico a otro. Un ejemplo se muestra en la Figura 8, donde el alumno debe identificar la variable representada y sus categorías y recordar el concepto de frecuencia, para leer en el gráfico la frecuencia de cada valor y traducirla a la tabla, incorporando igualmente el título y etiquetas de las categorías. 


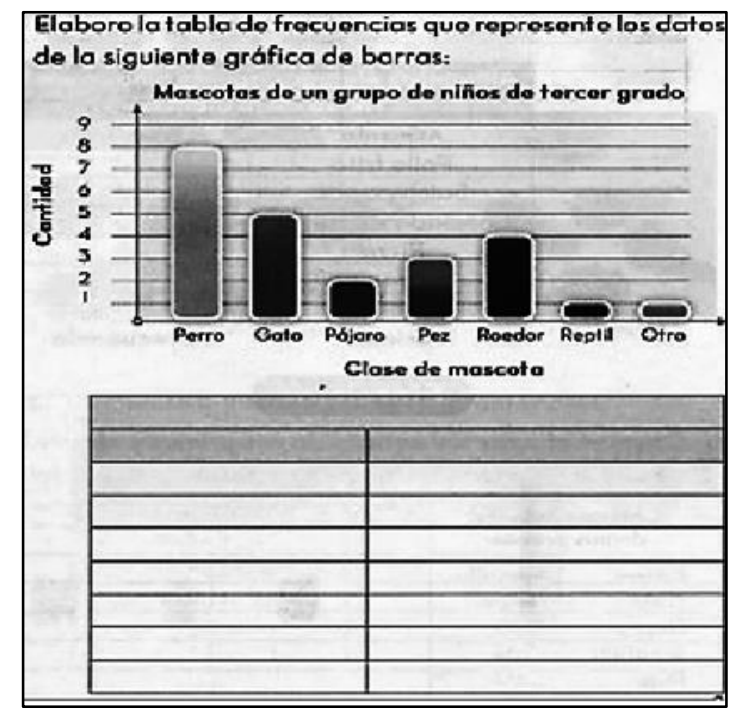

Figura 8 - Traducir a tabla Fuente: tomado de AL3 p. 158

Inventar problema: cuando se solicita, explícitamente, plantear una nueva situación a partir de los datos de una representación gráfica. Así, en la última pregunta de la Figura 9 se pide inventar una situación problemática que se justifique la construcción del gráfico.

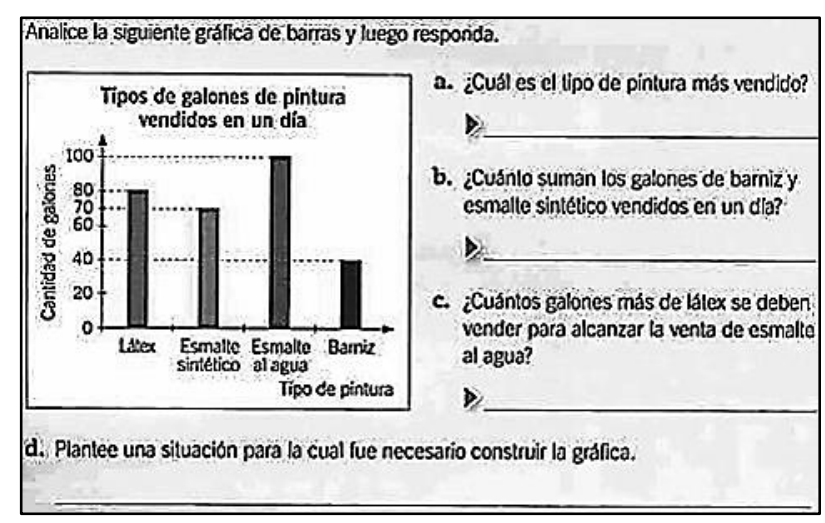

Figura 9 - Inventar problema

Fuente: tomado de S5, p. 238

En la Tabla 9 se muestra que el tipo de tarea que más se utiliza en las series de libros analizadas corresponde a la lectura del gráfico, seguido por el cálculo a partir de la información dada en el mismo. El resto de tareas tiene menor relevancia y en la mayoría se requieren la lectura y el cálculo, siendo poco frecuentes las actividades que piden traducir un gráfico o la invención de problemas.

En nuestro estudio hay una clara similitud con los libros de texto españoles y chilenos, según se describe en Díaz-Levicoy et al. (2016). La lectura representa el 49,8\% en los libros españoles, mientras que la actividad del cálculo representa el 52,2\% en el caso chileno. Por el contrario, la actividad de ejemplificar tiende a ser significativa; 16,7\% en España, $12,6 \%$ en Chile. Otra diferencia, en estos estudios, la presentan las actividades de construcción; en los libros españoles esta actividad representa $27,9 \%$, en Chile representa un $24,8 \%$, mientras en 
los libros costarricenses, la construcción representa solo un 5,5\% de las actividades analizadas.

Tabla 9 - Frecuencia y porcentaje de gráficos por tipo de tarea y editorial

\begin{tabular}{ccccc}
\hline & \multicolumn{4}{c}{ Editorial } \\
\cline { 2 - 5 } Tipo de Tarea & $\mathbf{N}$ & $\mathbf{A L}$ & $\mathbf{N}$ & $\mathbf{\%}$ \\
\cline { 2 - 5 } & $\mathbf{N}$ & 49,7 & 106 & 42,2 \\
Leer el gráfico & 73 & 8,2 & 16 & 6,4 \\
Completar & 12 & 20,4 & 86 & 34,3 \\
Calcular & 30 & 7,5 & 11 & 4,4 \\
Construir & 11 & 6,1 & 18 & 7,2 \\
Ejemplificar & 9 & 1,4 & 7 & 2,8 \\
Traducir a otra representación & 2 & 6,8 & 7 & 2,8 \\
Inventar problema & 10 & $\mathbf{1 0 0}$ & $\mathbf{2 5 1}$ & $\mathbf{1 0 0}$ \\
\hline Total & $\mathbf{1 4 7}$ &
\end{tabular}

Fuente: elaborado por los autores

Como se aprecia en la Tabla 10, la tarea de ejemplificar es utilizada en los niveles de segundo a sexto año, lo que también es destacado en el estudio de Díaz-Levicoy et al. (2016). La tarea de construir gráficos es impulsada con mayor frecuencia en cuarto y quinto, sin ser predominante, como si sucede en los libros españoles y chilenos.

Tabla 10 - Frecuencia y porcentaje de gráficos por tipo de tarea y año escolar

\begin{tabular}{|c|c|c|c|c|c|c|c|c|c|c|c|c|}
\hline \multirow{2}{*}{ Tipo de Tarea } & \multicolumn{2}{|r|}{$\mathbf{1}^{\circ}$} & \multicolumn{2}{|c|}{$2^{\circ}$} & \multicolumn{2}{|r|}{$3^{\circ}$} & \multicolumn{2}{|c|}{$4^{\circ}$} & \multicolumn{2}{|c|}{$5^{\circ}$} & \multicolumn{2}{|c|}{$6^{\circ}$} \\
\hline & $\mathbf{N}$ & $\%$ & $\mathbf{N}$ & $\%$ & $\mathbf{N}$ & $\%$ & $\mathbf{N}$ & $\%$ & $\mathbf{N}$ & $\%$ & $\mathbf{N}$ & $\%$ \\
\hline Leer el grafico & 18 & 56,3 & 36 & 40,9 & 35 & 41,8 & 36 & 44,4 & 30 & 44,1 & 36 & 48,6 \\
\hline Completar & 6 & 18,8 & 13 & 14,8 & 3 & 5,5 & 1 & 1,2 & 4 & 5,9 & 1 & 1,4 \\
\hline Calcular & 8 & 25 & 24 & 27,3 & 11 & 20,0 & 29 & 35,8 & 20 & 29,4 & 24 & 32,4 \\
\hline Construir & & & 3 & 3,4 & 3 & 5,5 & 6 & 7,4 & 6 & 8,8 & 4 & 5,4 \\
\hline Ejemplo & & & 9 & 10,2 & 5 & 9,1 & 5 & 6,2 & 4 & 5,9 & 4 & 5,4 \\
\hline $\begin{array}{l}\text { Traducir a otra } \\
\text { representación }\end{array}$ & & & & & 9 & 15,4 & & & & & & \\
\hline $\begin{array}{c}\text { Inventar } \\
\text { problema }\end{array}$ & & & 3 & 3,4 & 1 & 1,8 & 4 & 4,9 & 4 & 5,9 & 5 & 6,8 \\
\hline Total & 32 & 100,0 & 88 & 100,0 & 55 & 100,0 & 81 & 100,0 & 68 & 100,0 & 74 & 100,0 \\
\hline
\end{tabular}

\subsection{Propósito del gráfico dentro de la tarea}

El gráfico es considerado, según Kosslyn (1985), como un recurso que se utiliza para análisis, comunicación y en la enseñanza tradicional, para fines meramente constructivos. Esta variable no ha sido tenida en cuenta en investigaciones previas, por lo que estos resultados representan una contribución original, por lo que su clasificación se describe a continuación.

Propósito de análisis: en este caso, el uso principal del gráfico es descubrir o identificar características del conjunto de datos. Se trataría de fomentar el proceso de 
transnumeración, descrito por Wild y Pfannkuch (1999), que es uno de los procesos básicos de razonamiento estadístico y consiste en descubrir características del conjunto de datos no visibles en los datos sin organizar, mediante un cambio de representación de los mismos. Ejemplos de este propósito se presentan en la Figura 5 donde el estudiante ha de usar el gráfico para descubrir algunas características de la situación.

Propósito de comunicación: los gráficos clasificados en este tipo son aquellos utilizados con el fin de transmitir información sobre los datos y relaciones entre ellos. En este caso, el constructor del gráfico no precisa descubrir características de los datos, sino comunicarlas a otra persona en forma eficiente. Un ejemplo es la Figura 8, que pide traducir un gráfico a tabla - el propósito del gráfico aquí es comunicar la información requerida para construir la tabla; no se pide identificar ninguna información a partir del gráfico.

Propósito de construcción: adicionalmente a lo establecido en Kosslyn, (1985), añadimos el propósito constructivo, importante desde el punto de vista de la enseñanza tradicional, donde en algunas actividades el interés está centrado en que el estudiante aprenda la construcción del gráfico y no en el análisis o la comunicación de información. Un ejemplo se visualiza en la Figura 4, donde el objetivo es la construcción del gráfico.

Las actividades analizadas en los libros de texto tienden a utilizar los gráficos con propósito de análisis. Un 59,8\% del total de actividades se utiliza con este fin, mientras que solo el $25,4 \%$, son utilizados con propósito de comunicación, tal y como se observa en la Tabla 11. El propósito constructivo no parece ser tan relevante en ambos editoriales y solo representa el 14,8\% del total de actividades analizadas.

Tabla 11 - Frecuencia y porcentaje de gráficos por tipo de propósito y editorial

\begin{tabular}{ccccc}
\hline \multirow{2}{*}{ Tipo de propósito } & \multicolumn{4}{c}{ Editorial } \\
\cline { 2 - 5 } & $\mathbf{A L}$ & $\mathbf{N}$ & $\mathbf{N}$ & $\mathbf{\%}$ \\
\cline { 2 - 5 } & $\mathbf{N}$ & 55,8 & 156 & 62,2 \\
\cline { 2 - 5 } Análisis & 82 & 26,5 & 62 & 24,7 \\
Comunicación & 39 & 17,7 & 33 & 13,1 \\
Construcción & 26 & $\mathbf{1 0 0 , 0}$ & $\mathbf{2 5 1}$ & $\mathbf{1 0 0 , 0}$ \\
\hline Total & $\mathbf{1 4 7}$ & Fuente: elaborado por los autores
\end{tabular}

Analizando los resultados mostrados en la Tabla 12, se observa que el propósito en primer grado es el de comunicar información $(62,5 \%)$ y poco se incentiva el análisis y la construcción de gráficos. Por su parte, en los demás grados escolares predomina el propósito de análisis con un 59,8\% de las actividades catalogadas, y solo en tercer año se muestra un leve interés por la construcción gráfica. 
Tabla 12 - Frecuencia y porcentaje de gráficos por tipo de propósito y año escolar

\begin{tabular}{ccccccccccccc}
\hline & \multicolumn{10}{c}{ Año Escolar } \\
\cline { 2 - 12 } Tipo de propósito & \multicolumn{1}{c}{$\mathbf{1}^{\circ}$} & \multicolumn{1}{c}{$\mathbf{2}^{\circ}$} & $\mathbf{4}^{\circ}$ & \multicolumn{1}{c}{$\mathbf{5}^{\circ}$} & $\mathbf{6}^{\circ}$ \\
\cline { 2 - 12 } & $\mathbf{N}$ & $\mathbf{\%}$ & $\mathbf{N}$ & $\mathbf{\%}$ & $\mathbf{N}$ & $\mathbf{\%}$ & $\mathbf{N}$ & $\mathbf{\%}$ & $\mathbf{N}$ & $\mathbf{\%}$ & $\mathbf{N}$ & $\mathbf{\%}$ \\
\cline { 2 - 11 } & 6 & 18,8 & 37 & 42 & 35 & 63,6 & 56 & 69,1 & 47 & 69,1 & 57 & 77 \\
Análisis & 20 & 62,5 & 35 & 39,8 & 7 & 12,7 & 16 & 19,8 & 10 & 14,7 & 13 & 17,5 \\
Comunicación & 6 & 18,8 & 16 & 18,2 & 13 & 23,6 & 9 & 11,1 & 11 & 16,2 & 4 & 5,4 \\
Construcción & $\mathbf{3 2}$ & $\mathbf{1 0 0 , 0}$ & $\mathbf{8 8}$ & $\mathbf{1 0 0 , 0}$ & $\mathbf{5 5}$ & $\mathbf{1 0 0 , 0}$ & $\mathbf{8 1}$ & $\mathbf{1 0 0 , 0}$ & $\mathbf{6 8}$ & $\mathbf{1 0 0 , 0}$ & $\mathbf{7 4}$ & $\mathbf{1 0 0 , 0}$ \\
\hline Total & \multicolumn{10}{c}{ Fuente: elaborado por los autores }
\end{tabular}

\section{Conclusiones}

El análisis de los gráficos estadísticos en los libros de texto en las dos editoriales de uso más difundido en Costa Rica proporciona información sobre cómo es introducido este elemento en la educación estadística y complementa los estudios realizados en otros países. Se ha mostrado un predominio del gráfico de barras, en coincidencia con el estudio de DíazLevicoy et al. (2016) y con las recomendaciones establecidas en las orientaciones curriculares en Costa Rica, España y Chile (MEC, 2006; M.E.P, 2012; MINEDUC, 2012). Adicionalmente, se incorporan diagramas de puntos y gráficos de sectores de manera paulatina, pasando del gráfico de barras, pictogramas y diagramas de puntos en el primer ciclo, a gráficos circulares y de líneas o barras múltiples en el segundo ciclo de la educación primaria. Esta estrategia es coherente con las orientaciones curriculares costarricenses.

Los textos analizados hacen uso frecuente de actividades donde se requiere un nivel 2 de lectura; es decir leer dentro de los datos, pero también de los niveles 1 y 3 , lo que coincide con la recomendación de estimular el análisis e interpretación de la información que comunican los datos, propuesto en las orientaciones curriculares. El hecho de que los libros de texto analizados en Costa Rica brinden un espacio importante a las actividades de nivel 3, leer más allá de los datos, refleja una diferencia importante con respecto a los resultados españoles obtenidos en Díaz-Levicoy et al. (2016), donde este nivel es poco frecuente.

La complejidad semiótica del gráfico suele ser de nivel 3 de representación de una distribución de datos, lo que también coincide con los estudios en Díaz-Levicoy et al. (2016) y el nivel semiótico de los gráficos avanza en complejidad conforme se progresa en los años escolares.

La tarea más común corresponde a leer, calcular y completar, lo que concuerda con los lineamientos curriculares costarricenses y en concordancia con Díaz-Levicoy et al. (2016). Se siguen las recomendaciones de la NCTM (2000) en cuanto a promover el análisis y reflexión en la búsqueda de inferencias y predicciones a través de los datos. Si bien en las orientaciones 
curriculares en Costa Rica, la construcción de gráficos es propuesta formalmente a partir de cuarto año, las editoriales analizadas introducen tareas del tipo constructivas desde el segundo año escolar.

Un aspecto que no ha quedado reflejado en los tipos de tarea corresponde al interés ministerial de promover la resolución de problemas y el uso de tecnologías digitales como ejes transversales de las matemáticas. Por otro lado, los lineamientos curriculares enfatizan en la necesidad de que el estudiante sea capaz de identificar posibles errores en los datos recolectados, tarea que no fue posible identificar en las dos series de libros analizadas.

En cuanto al propósito del gráfico se asume una línea de actividades de análisis y de comunicación, lo cual se alinea con los intereses del Ministerio de Educación Pública de Costa Rica, en promover el análisis e interpretación de la información a partir de los datos; sin embargo, al realizar el estudio por año escolar, actividades con propósito constructivo aparecen desde el segundo año y constituye el segundo propósito más importante en el tercer año.

En el contexto en el que se desarrollan los gráficos, se encuentra que el contexto escolar, es el más frecuente, reflejando coherencia con lo establecido en las recomendaciones curriculares dadas en (M.E.P, 2012), donde se indica la necesidad de realizar el estudio de los gráficos estadísticos con temas cercanos al estudiante. El contexto varía conforme se avanza en los niveles educativos; en primer año, el contexto social y personal son los más frecuentes, pasando a ser lo laboral /escolar el contexto más frecuente de las actividades de segundo a quinto año con un incremento significativo del contexto científico de cuarto a sexto año, donde se conveniente en el tema más frecuente en este último nivel.

En consecuencia, se observa una rica gama de actividades relacionadas con los gráficos estadísticos en los textos analizados, que se adaptan casi por completo a las orientaciones curriculares, con la excepción citada de uso de la tecnología y resolución de problemas. Estos serían los puntos en que el profesor podría insistir, para paliar su carencia y que deberían ser tenidos en cuenta en nuevas reediciones de los textos.

Toda esta información es útil al profesor, que puede tener en cuenta las variables que caracterizan las actividades con gráficos estadísticos y los valores más adecuados de las mismas para cada nivel educativo en su enseñanza del tema. El formador de profesores podría usar también nuestros resultados, para proponer actividades de análisis de texto a los futuros profesores y comprobar que tienen la formación adecuada, tanto para realizar este análisis, como para desarrollar la enseñanza. 


\section{Agradecimiento}

Proyecto PID2019-105601GB-I00 (AEI) y Grupo FQM126 (Junta de Andalucía)

\section{Referencias}

AL1. CALDERÓN, Y. (Ed.). Matemática 1. San José, Costa Rica: Asociación Libros para Todos. 2017.

AL2. CALDERÓN, Y. (Ed.). Matemática 2. San José, Costa Rica: Asociación Libros para Todos. 2017.

AL3. CALDERÓN, Y. (Ed.) Matemática 3. San José, Costa Rica: Asociación Libros para Todos. 2017.

AL4. CALDERÓN, Y. (Ed.) Matemática 4. San José, Costa Rica: Asociación Libros para Todos. 2017.

AL5. CALDERÓN, Y. (Ed.) Matemática 5. San José, Costa Rica: Asociación Libros para Todos. 2017.

AL6. CALDERÓN, Y. (Ed.) Matemática 6. San José, Costa Rica: Asociación Libros para Todos. 2017.

ARTEAGA, P. Evaluación de conocimientos sobre gráficos estadísticos y conocimientos didácticos de futuros profesores. 2011. 403f. Tesis (Doctorado en Didáctica de la Matemática) Departamento de Didáctica de la Matemática, Universidad de Granada, Granada, 2011.

ARTEAGA, P.; BATANERO, C. Relating graph semiotic complexity to graph comprehension in statistical graphs produced by prospective teachers. En: Seventh Congress of the European Society For Research in Mathematics Education, Rzeszów. Proceedings... Rzeszów, Polonia: ERME, 2011, p. 368-377.

BATANERO, C. (2013). Sentido estadístico: Componentes y desarrollo. In: JORNADAS VIRTUALES EN DIDÁCTICA DE LA ESTADÍSTICA, PROBABILIDAD Y COMBINATORIA, 2013, Granada. Actas... Granada: Grupo de Investigación FQM-126, 2013, p. 55-61.

BATANERO, C.; ARTEAGA, P.; RUIZ, B. Análisis de la complejidad semiótica de los gráficos producidos por futuros profesores de educación primaria en una tarea de comparación de dos variables estadísticas. Enseñanza de las Ciencias, Barcelona, v. 28. n. 1, p. 141-154, marzo, 2010.

BATANERO, C.; DÍAZ, C; CONTRERAS, M.; ROA, R. El sentido estadístico y su desarrollo. Números, La Laguna v. 83, n.1, p. 7-18, jul. 2013.

BERTIN, J. Semiologie graphique. Paris: Gauthier-Villars, 1967.

BIVAR, D.; SELVA, A. Analisando atividades envolvendo gráficos e tabelas nos livros didáticos de matemática. In: CONFERÊNCIA INTERAMERICANA DE EDUCAÇÃO MATEMÁTICA (XIII CIAEM), 2011, Recife. Actas... Recife: Comité Interamericano de Educación Matemática, 2011. Disponible en: http://xiii.ciaem-redumate.org/index.php/xiii_ciaem/xiii_ciaem/paper/view/1332. Acceso en: 18 mar 2020. 
CASTELLANOS, M. Tablas y gráficos estadísticos en pruebas SABER - Colombia. 2013.

Disertación (Máster en Didáctica de la Matemática) - Departamento de Didáctica de la Matemática, Universidad de Granada, Granada, 2013.

CAZORLA, I. A relação entre as habilidades viso-pictóricas eo domínio de conceitos estatísticos na leitura de gráficos. 2002. Tese (Doutorado em Educação) - Universidade Estadual de Campinas, Campinas, 2002.

CCSSI. Common Core State Standards for Mathematics. Washington: National Governors Association for Best Practices and the Council of Chief State School Officers, 2010.

CURCIO, F. R. Comprehension of mathematical relationships expressed in graphs. Journal for Research in Mathematics Education, Reston, v.5, n.18, p.382-393, 1987.

CURCIO, F. R. Developing graph comprehension. Reston: NCTM, 1989.

DÍAZ-LEVICOY, D.; ARTEAGA, P. Análisis de gráficos estadísticos en textos escolares de séptimo básico en Chile. Revista Electrónica Diálogos Educativos, Santiago de Chile, v.14, n.28, 21-40, nov. 2014.

DÍAZ-LEVICOY, D. et al. Gráficos estadísticos en libros de texto de Educación Primaria: un estudio comparativo entre España y Chile. Bolema, Rio Claro (SP), v.30, n. 55, p.713-737, ago. 2016.

DÍAZ-LEVICOY, D.; GIACOMONE, B.; ARTEAGA, P. Caracterización de los gráficos estadísticos en libros de texto argentinos del Segundo Ciclo de educación primaria. Profesorado, Granada, v.21, n. 3, 299-326, sep. 2017.

ENGEL, J. Statistical literacy and society. What is civic statistics? In: CONGRESO

INTERNATIONAL VIRTUAL DE EDUCACIÓN ESTADÍSTICA, 3, 2019, Actas... Granada: Grupo de Investigación de Educación Estadística, 2019. Disponible en:

https://www.ugr.es/ fqm126/civeest/ponencias/engel.pdf. Acceso en: 18 mar 2020.

FRIEL, S.; CURCIO, F.; BRIGHT, G. Making sense of graphs: Critical factors influencing comprehension and instructional implications. Journal for Research in Mathematics Education, Reston, v. 32, n. 2, p. 124-158, may. 2001.

GAL, I. Adults' statistical literacy: meanings, components, responsibilities. International Statistical Review, La Haya, v. 70, n. 1, p. 1-25, ene. 2002.

HERBEL, B. A. From intended curriculum to written curriculum: Examining the "voice" of a mathematics textbook. Journal for Research in Mathematics Education, Reston, v. 38, n. 4, p. 344369, 2007.

KOSSLYN, S. M. Graphics and human information processing. Journal of the American Statistical Association, Alexandria, v. 80, n. 3, p. 499-512, sep.1985.

LEMOS, M. O estudo do tratamento da informação nos livros didáticos das séries iniciais do Ensino Fundamental. Ciência e Educação, Sao Paulo, v. 12, n. 2, p. 171-184, jun. 2006.

Ministerio de Educación Pública - M.E.P. Programa de Estudio Matemáticas, I, II y III Ciclos de la Educación General Básica y Ciclo Diversificado. San José: Ministerio de Educación Pública, 2012. 
MINISTERIO DE EDUCACIÓN, CULTURA Y DEPORTE - MECD. Real Decreto 126/2014, de 28 de febrero, por el que se establece el currículo básico de la educación primaria. Madrid: MECD, 2014.

MINISTERIO DE EDUCACIÓN Y CIENCIA - MINEDUC. Matemática educación básica. Bases curriculares. Santiago: Unidad de Currículum y Evaluación, 2012.

MINGORANCE, C. La estadística en las pruebas de diagnóstico andaluzas. 2014. Trabajo fin de Grado (Grado en Educación Primaria) - Departamento de Didáctica de la Matemática, Universidad de Granada, Granada, 2014.

NATIONAL COUNCIL OF TEACHERS OF MATHEMATICS. Principles and standards for school mathematics. Reston: NCTM, 2000.

ORGANIZATION FOR ECONOMIC COOPERATION AND DEVELOPMENT - OECD. PISA 2015 assessment and analytical framework: Science, reading, mathematic and financial literacy. Paris: OECD Publishing, 2016.

PORTA, L. Y SILVA, M. La investigación cualitativa: el análisis de contenido en la investigación educativa. Córdoba: CENDIE, 2003.

RIDGWAY, J. Implications of the data revolution for statistics education. International Statistical Review, La Hayam v. 84, n. 3, p. 528-549, sep. 2016.

S1. SANTILLANA. Matemática 1. San José, Costa Rica: Santillana. 2016.

S2. SANTILLANA. Matemática 2. San José, Costa Rica: Santillana. 2016.

S3. SANTILLANA. Matemática 3. San José, Costa Rica: Santillana. 2016.

S4. SANTILLANA. Matemática 4. San José, Costa Rica: Santillana. 2016.

S5. SANTILLANA. Matemática 5. San José, Costa Rica: Santillana. 2016.

S6. SANTILLANA. Matemática 6. San José, Costa Rica: Santillana. 2016.

SHAUGHNESSY, J. M.; GARFIELD, J.; GREER, B. Data handling. In: BISHOP, A. J.; CLEMENTS; K.; KEITEL, C.; KILPATRICK, J.; LABORDE, C. (Org.). International handbook of mathematics education. Dordrecht: Kluwer Academic Publishers, 1996, p. 205-237.

WATSON, J. M. Statistical literacy at school: Growth and goals. Mahwah: Lawrence Erlbaum, 2006.

WILD, C.; PFANNKUCH, M. Statistical thinking in empirical enquiry. International Statistical Review, La Haya, v. 6, n. 3, p. 223-265, sep. 1999.

Submetido em 23 de Abril de 2019. Aprovado em 09 de Setembro de 2019. 